\title{
Aplicación integrada a la tecnología Kinect para el reconocimiento e interpretación de la Lengua de Señas Colombianas
}

\section{Integrated application for Kinect technology for recognition and interpretation of Colombian Sign Language}

\author{
Esmeide Leal Narváez
}

Magister en Ingeniería de Sistemas énfasis en Sistemas Docente Ingeniería de Sistemas

Universidad Autónoma del Caribe, Email: esleal@uac.edu.co

Nallig Leal Narváez

Magister en Ingeniería de Sistemas énfasis en Sistemas Docente Ingeniería de Sistemas Universidad Autónoma del Caribe,Email: nleal@uac.edu.co

\section{Carlos Henríquez Miranda}

Magister en Ingeniería de Sistemas y computación, Docente Ingeniería de Sistemas Universidad Autónoma del Caribe, Email: chenriquez@uac.edu.co

\section{Leydi Patricia Pichón Pacheco}

Tecnóloga en Informática. Estudiante Universidad Autónoma Del Caribe, Email: leydi.p19@gmail.com.

\section{Shirley Paola Romero Martínez}

Tecnóloga en Informática. Estudiante Universidad Autónoma Del Caribe, Email: hirley.930510@ gmail.com

Para Citar este Artículo: Leal, E., Leal N., Henríquez, C. (2016). Aplicación integrada a la tecnología Kinect para el reconocimiento e interpretación de la Lengua de Señas Colombianas.Escenarios, 14 (2), p,p 7-19 DOI: http: / / dx.doi.org/10.15665/ esc.v14i2.928

Recibido: agosto 2-2016

Aceptado: septiembre 92016

\section{RESUMEN}

SEn Colombia, el tema de inclusión social se ha convertido en uno de los pilares importantes en el país, considerando a las personas que poseen deficiencia para realizar sus actividades cotidianas. Hoy en día, los avances tecnológicos han ayudado a disminuir estas deficiencias y han mejorado significativamente la calidad de vida de estas personas, como lo es el uso de aplicaciones de autoaprendizaje braille, herramientas de entrenamiento fonético, aplicaciones de reconocimiento de señales, entre otras. Este artículo presenta el desarrollo de una aplicación capaz de fortalecer las estrategias didácticas de las instituciones educativas referentes a la enseñanza de la lengua de señas colombiana. Esta herramienta permite a los estudiantes aprender las señas básicas por medio de un glosario gráfico, siendo evaluada la ejecución de las mismas capturando la escena por medio de un sensor de profundidad que ofrece la tecnología Kinect; con la imagen captada se compara con una base de datos de señas para determinar si ésta se realiza de manera correcta y así asignar un puntaje de evaluación. De igual manera, el uso de la tecnología Kinect en esta aplicación motiva a los estudiantes a aprender el lenguaje, dado que dicha tecnología brinda facilidades y una dinámica de aprendizaje más acorde con el desarrollo de las nuevas metodologías de educativas. Se implementó en los grupos de transición en instituciones educativas que prestan servicio de educación a los estudiantes con discapacidad auditiva; esta población es muy importante debido a que es la oportu- 
nidad de comenzar a construir bases sólidas en el conocimiento de la lengua de señas. Los resultados muestran que esta aplicación tiene aceptación y cumple con el objetivo propuesto.

Palabras Clave: Kinect, Lengua de Señas, C\#, Sordomudo, Accesibilidad, PostgreSQL.

\begin{abstract}
Social inclusion in Colombia is very important, so consideration is given to people who present some type of limitation to carry out their daily activities. Current technological advances can help reduce these limitations and thus improve the quality of life of these people. These advances include Braille self-learning applications, phone training tools and sign-in applications, among others. This article presents the development of an application capable of strengthening the didactic strategies of educational institutions involved in the teaching of Colombian sign language. This tool allows students to learn the basic signs by means of a graphic glossary, which is evaluated by capturing the scene using a depth sensor offered by Kinect technology. The captured image is compared to a sign database to determine if it is performed correctly and thus assigns an evaluation score; similarly, the use of Kinect technology in this application motivates students to learn the language, since this technology provides facilities and learning dynamics more in line with the development of new educational methodologies. This tool was implemented in transition groups in educational institutions that provide education services to hearing impaired students. This population is very important because it is the opportunity for beginning to build solid foundations in the knowledge of sign language. The results show that this application has acceptance and meets the proposed objective.
\end{abstract}

Key words: Kinect, Sign Language, C \#, Deaf Mute, Accessibility, PostgreSQL.

\title{
INTRODUCCIÓN
}

Uno de los derechos fundamentales de los niños es la educación, tal como lo señala el Artículo 67 de la Constitución Política de Colombia (Constitución Colombia, 1991). Por su parte, la Convención Internacional sobre los Derechos de las Personas con Discapacidad señala en el Artículo 24, que los Estados deben hacer efectivo este derecho sobre la base de la igualdad de oportunidades. (UN, 2006)

En los últimos años, el Gobierno Colombiano ha impulsado grandes cambios sobre la comunidad sordomuda, lo cual ha permitido garantizar las oportunidades de inclusión en la sociedad. A través del desarrollo de la ley 324 de 1996 (Ley324, 1996), se reconoce la lengua de señas como lengua nativa de las personas con discapacidad auditiva, al igual que su estilo y necesidades comunicativas; sin embargo, esto no ha sido suficiente, debido a que muchas de estas personas no la han podido desarrollar efectivamente, por el difícil acceso que tienen a los recursos en la escuelas.

Las instituciones educativas que promuevan y fortalezcan el uso de la lengua de señas en los niños y niñas de su comunidad, incluidos aquellos que tienen algún tipo de discapacidad, requieren una transformación significativa en la atención y la propuesta pedagógica, para atender las necesidades educativas de sus estudiantes, su principal objetivo se debe centrar en eliminar las barreras de comunicación. Para que las comunidades educativas y los docentes puedan responder a este desafío es necesario proporcionarles ayuda y apoyo sostenido, a través de actividades de formación, asesoramiento y herramientas de aprendizaje. (Ministerio de Educación Nacional, 2006, pág. 3)

En la actualidad las instituciones educativas que acogen a personas con discapacidad auditiva cuentan con pocas herramientas que les faciliten 
a estas el aprendizaje de las lenguas de señas, debido a que dichas herramientas presentan un alto costo y las instituciones no cuentan con los recursos necesarios para ser adquiridas; por lo cual se han realizado investigaciones encaminadas a mejorar este percance, así Talledo, Villegas y Barrientos (2015), proponen una serie de proyectos que mejoran radicalmente la independencia y estilo de vida de las personas con discapacidad mediante el empleo de tecnologías y soluciones existentes. Ellos describen una forma de resolver la limitación comunicativa de los sordomudos empleando Creative, una cámara interactiva gestual, en un sistema de reconocimiento de señales; una aplicación de entrenamiento fonético como tratamiento para la pos-cirugía del labio leporino, una aplicación móvil para una mayor accesibilidad de los ciegos, y una aplicación de autoaprendizaje braille con un teclado orientado para ciegos.

Así mismo, Cujano y Vera (2016) desarrollan un sistema interactivo mediante tecnología Kinect para desarrollar habilidades psicomotrices en personas con discapacidad visual. Kinect Move, nombrado así el sistema interactivo, tiene cinco actividades para desarrollar la psicomotricidad, programadas en el lenguaje de programación C\# de Visual Studio, este es capaz de reconocer al esqueleto del usuario en cualquier ambiente que desee realizar las actividades e interactuar con él por medio de comandos de voz y audio, recalcando que para mejorar esta herramienta se recomienda la utilización de filtros, los cuales capten únicamente la voz del usuario, ya que el Kinect no es un sensor inteligente y tiende a confundir los datos que recibe.

En este sentido Castro (2013), presenta hallazgos que aportan evidencia a favor de la incorporación temprana de los niños sordos al aprendizaje de lenguaje de señas. Para esto proporciona información desde la descripción del proceso de adquisición lingüística en niños sordos, hijos de madres sordas y otros relativos a las ventajas, en términos académicos y cognitivos, de niños sordos que aprenden tempranamente lenguaje de señas y posibles consecuencias en su desarrollo cognitivo y social. Entonces, ante la necesidad de discutir la conveniencia del aprendizaje temprano de la lengua de señas (LS) por parte de los niños sordos, realiza una revisión de investigaciones recientes que permiten apoyar la incorporación temprana de LS en niños sordos hijos de oyentes. Para ello describe en primer lugar estudios que muestran principalmente las similitudes entre el proceso de adquisición del lenguaje de señas en niños sordos, y el de lenguaje oral en niños oyentes. Luego, destaca la importancia una comunicación temprana efectiva entre padres oyentes e hijos sordos, para el desarrollo lingüístico, psicológico y social de estos niños. Finalmente, describen estudios que muestran los beneficios del aprendizaje del LS para el desarrollo de competencias efectivas de comunicación en los niños sordos, incluida el habla oral, y para el desarrollo de estos niños en el ámbito académico y cognitivo social.

Así, Parra (2015) presenta una herramienta computacional basada en procesamiento de imágenes para asistir a las personas que presentan discapacidad auditiva. La herramienta está en capacidad de capturar una secuencia de imágenes que corresponde a los gestos que el usuario hace con sus manos para realizar alguno de los signos empleados para la comunicación no verbal. La herramienta somete esta información a una serie de etapas de procesamiento con miras a identificar apropiadamente de qué gesto se trata y a emitir su equivalente textual. Los resultados obtenidos muestran que se logró identificar correctamente y en diversidad de situaciones los signos correspondientes al lenguaje de signos americano, el más empleado a escala mundial y base del lenguaje colombiano.

De igual forma, Rodríguez, Pineda y Sánchez (2013) desarrollan un prototipo capaz de tomar los puntos de una escena captada por medio del sensor de profundidad de Kinect, aplicar un filtro para eliminar datos innecesarios y posteriormente mostrar la Mesh que reconstruye la imagen de la escena final en $2 \mathrm{D}$, estableciendo la diferencia de distancias con el cambio de color. La herramienta detecta la localización de la o las manos en el Mesh, luego toma imágenes del gesto manual y las compara con imágenes almacenadas en archivo; estas cuentan con la traducción respectiva. Si encuentra una imagen con 
alto grado de coincidencia se devuelve el texto correspondiente.

Igualmente López, et al (2015) desarrollan una interfaz de reconocimiento de movimientos para el lenguaje de señas mexicano implementando el Kinect, ocupando principalmente palabras y el abecedario, utilizando los movimientos más comunes para desarrollar dicha interfaz interactiva que traduzca palabras del lenguaje de señas mexicano al idioma español, usando la tecnología del Kinect de Microsoft y empleando la metodología Scrum para el desarrollo de ésta, teniendo en cuenta que es ágil y flexible para gestionar el desarrollo de software.

Teniendo en cuenta lo anterior, este artículo tiene como finalidad mostrar el desarrollo de una herramienta interactiva para las personas con discapacidad auditiva, para que desde su primera etapa de aprendizaje, fortalezcan el idioma, y se facilite la traducción a la población sin ningún tipo de discapacidad o a personas que no tienen conocimiento de este lenguaje. Con esto se requiere reducir un poco la barrera que existe actualmente entre las personas con discapacidad auditiva y la sociedad, de manera que pueda ser comprensible la comunicación entre ellos.

Por otra parte, para el enfoque académico que se ofrece, se mostrarán por medio de videos los gestos del lenguaje al aprendiz, quien será evaluado capturando de la misma forma por medio del Kinect.

Para el desarrollo de la herramienta se realizó un análisis costo-beneficio con ayuda de parámetros importantes que influyen directamente en el proceso, tales como diseño, hardware, desarrollo, tecnología adicional a implementar, entre otros; con lo cual se determinó que desarrollar una aplicación integrada a la tecnología Kinect para el reconocimiento e interpretación del lenguaje signado colombiano, mejoraría significativamente el aprendizaje de los niños de temprana edad con discapacidad auditiva, permitiendo reforzar los conocimientos transmitidos por sus docentes. Además, el uso de la tecnología Kinect revolucionaria totalmente los métodos de aprendizaje impartidos en el sistema de educación colombiana para personas con discapacidad auditiva.

\section{Metodología}

El proyecto fue desarrollado para los niños de transición de la Institución Educativa Inobasol de Soledad - Atlántico con el fin de fortalecer desde su temprana edad las bases de la lengua de señas. Dentro de este contexto, se hace necesario desarrollar una aplicación de escritorio con tecnología Kinect que reconozca e interprete el lenguaje de señas colombianas para los estudiantes de transición, que se podrá desplegar en las siguientes etapas (Figura 1).

\section{Etapa 1: Recolección de la Información}

Esta etapa es fundamental en este proceso de diseño y desarrollo de la aplicación, por lo cual se seleccionó la entrevista como principal medio para la recolección de requisitos, debido a que con ésta se puede llegar a conocer a fondo las expectativas y opiniones de los entrevistados sobre el tema de la enseñanza de la lengua de señas a los estudiantes con discapacidad auditiva, y de esta forma conocer las necesidades y falencias en cuanto a recursos didácticos disponibles para facilitar la enseñanza a los niños. Además, se eligió como fuente primaria para este proyecto una encuesta estructurada. Este instrumento ha sido elegido por ser el más preciso al momento de tratar de ver los resultados para el desarrollo de este mismo.

\section{Etapa 2: Análisis de la Información}

Cabe resaltar que los entrevistados fueron los docentes, estudiantes e intérpretes de la Institución Educativa Inobasol, los cuales brindaron información específica para llevar a cabo el desarrollo del proyecto. Se trataron temas como las limitaciones a la hora de enseñar la lengua de señas, necesidades de los docentes para fortalecer la enseñanza de la lengua, expectativas sobre el proyecto a desarrollar, señas básicas, comunes y más usadas. Con dichos resultados se realizó un análisis estadístico con el fin de determinar si la propuesta de proyecto es realmente necesaria para ser implementada en la institución.

Donde a las personas encuestadas se les pidió que indicaran si les parecía conveniente que se utilice una aplicación tecnológica para reforzar el aprendizaje de las lenguas de señas desde 
Figura 1. Etapas del Proyecto

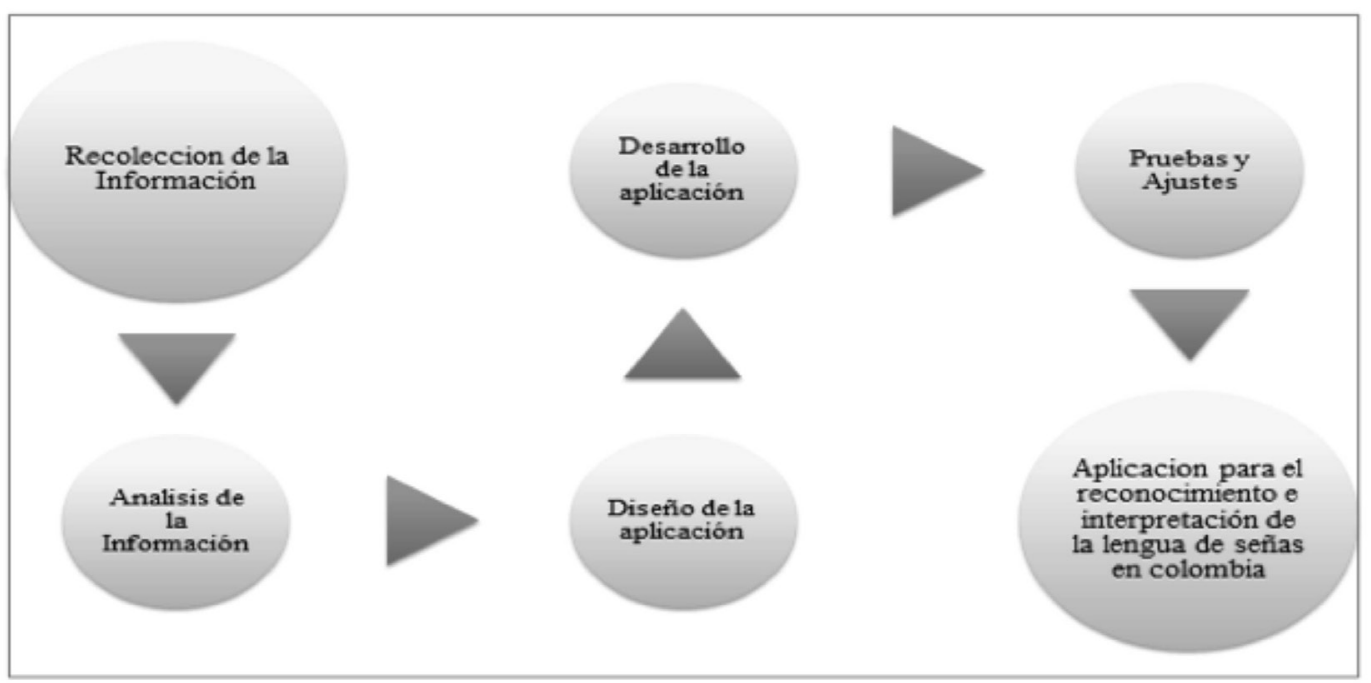

Fuente: Autores

temprana edad. El $197 \%$ está de acuerdo con la utilización de este tipo de iniciativa.

También se obtuvo una caracterización de los grupos de señas básicas más importantes para el aprendizaje de la lengua en los estudiantes de transición, aquí fueron priorizadas por las personas encuestadas; la Tabla 1 muestra el resultado a esta etapa.

De la anterior, se seleccionaron las categorías letras y números, para realizar el prototipo de la herramienta propuesta, de esta forma, se deter- minó que en la aplicación se reconocerán estas señas.

Etapa 3: Diseño de la aplicación

Una vez analizada y organizada toda la información recolectada en las fases anteriores, se determinó que información era necesaria para el diseño de la aplicación; aquí se logró determinar cada una de las partes y funciones con que contaría la herramienta. En la Figura 2 se muestra el diagrama de clases que representa la información que se obtiene del problema.

Tabla 1. Caracterización de Señas

\begin{tabular}{|l|c|}
\hline \multicolumn{1}{|c|}{ Categorías } & Resultado \\
\hline Letras (a, e, i, o, u, m, p, d, b) & $24 \%$ \\
\hline Saludos (Hola, adiós, ¿cómo estás?) & $13 \%$ \\
\hline Colores (Amarillo, azul, rojo) & $13 \%$ \\
\hline $\begin{array}{l}\text { Miembros de la Familia (Mama, papa, } \\
\text { hermano, tío) }\end{array}$ & $11 \%$ \\
\hline Números (1, 2, 3, 4, 5) & $7 \%$ \\
\hline $\begin{array}{l}\text { Días de la semana (Lunes, martes, } \\
\text { miércoles, jueves, viernes) }\end{array}$ & $7 \%$ \\
\hline Meses (Enero, febrero, marzo) & $7 \%$ \\
\hline
\end{tabular}


De la anterior, se seleccionaron las categorías letras y números, para realizar el prototipo de la herramienta propuesta, de esta forma, se determinó que en la aplicación se reconocerán estas señas.

Etapa 3: Diseño de la aplicación

Una vez analizada y organizada toda la información recolectada en las fases anteriores, se determinó que información era necesaria para el diseño de la aplicación; aquí se logró determinar cada una de las partes y funciones con que contaría la herramienta. En la Figura 2 se muestra el diagrama de clases que representa la información que se obtiene del problema.

Adicionalmente, la seguridad de la información es el centro de toda organización, por lo cual es importante resaltar que a medida que crece el personal, es mayor el número de información que se tiene que manejar; ante esto, se propone el diseño y modelado de un sistema de base de datos para almacenar la información que maneje la aplicación, la Figura 3 muestra el modelo relacional del sistema.

El siguiente diagrama de secuencia en la Figura 4 ilustra el comportamiento de cada una de las entidades de la herramienta. En este se puede percibir la forma como los usuarios interactua- ran con la aplicación.

Al terminar con el desarrollo de la aplicación, se realizaron respectivas pruebas, para detectar cualquier tipo de falla en el funcionamiento del sistema tecnología Kinect que reconozca e interprete el lenguaje de señas colombianas para los estudiantes de transición. Gracias a esto se realizaron pruebas unitarias, de integración, de sistema, regresión, instalación, análisis, funciones y usuarios; con el fin de verificar la funcionalidad y desempeño de cada una de las opciones de la aplicación.

Etapa 5: Pruebas y Ajustes

Inicialmente se realizarán las pruebas del sistema, donde se verificarán y corregirán los errores encontrados en los módulos, métodos, clases y objetos. A partir de allí, se comienza con la etapa de las pruebas de validación de requerimientos más conocida como pruebas de aceptación. Estas pruebas se enfocan en las acciones que realiza el usuario además de las salidas del sistema que puedan ser reconocidas por él, teniendo en cuenta los requisitos funcionales y no funcionales identificados para verificar la facilidad de uso del software desarrollado, eficiencia, entre otros, con lo que se pretende lograr la corrección de todo tipo de errores.

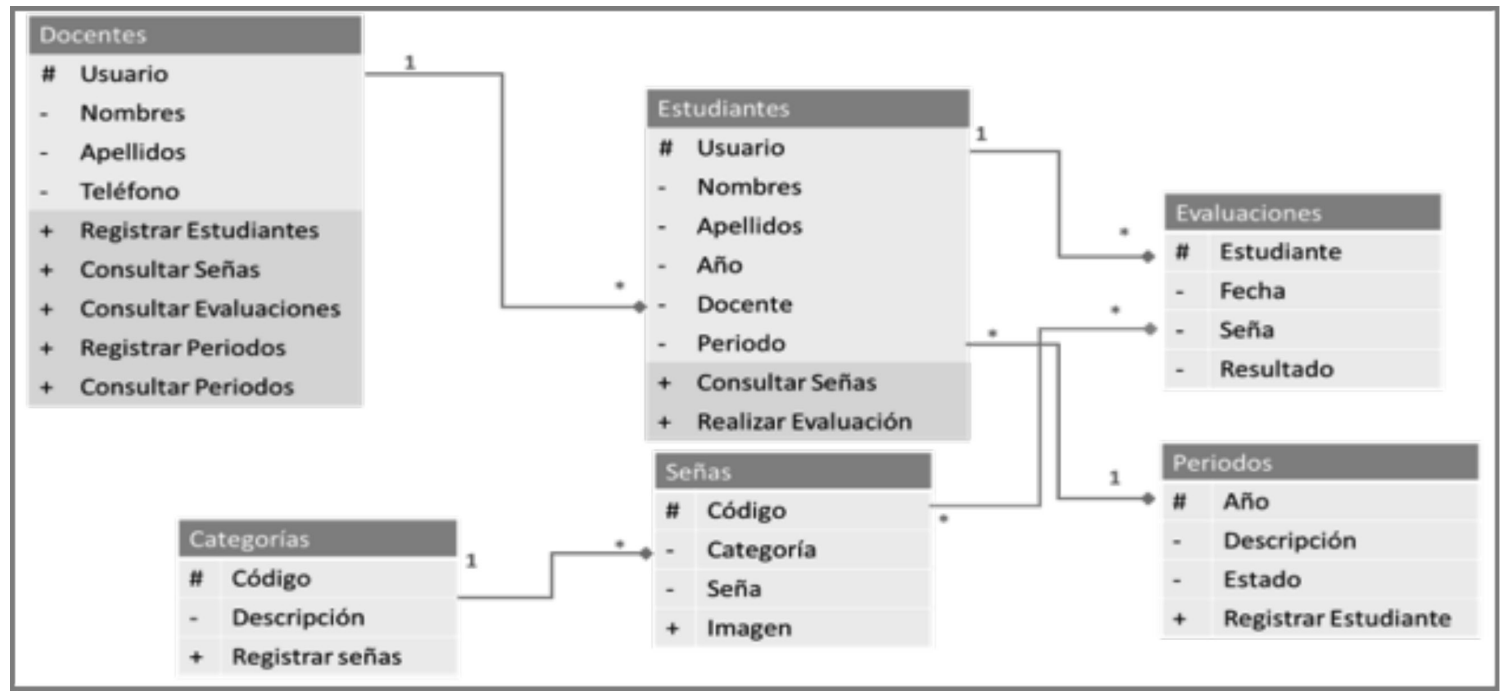

Fuente: Autores 
Figura 3. Modelo Entidad - Relación

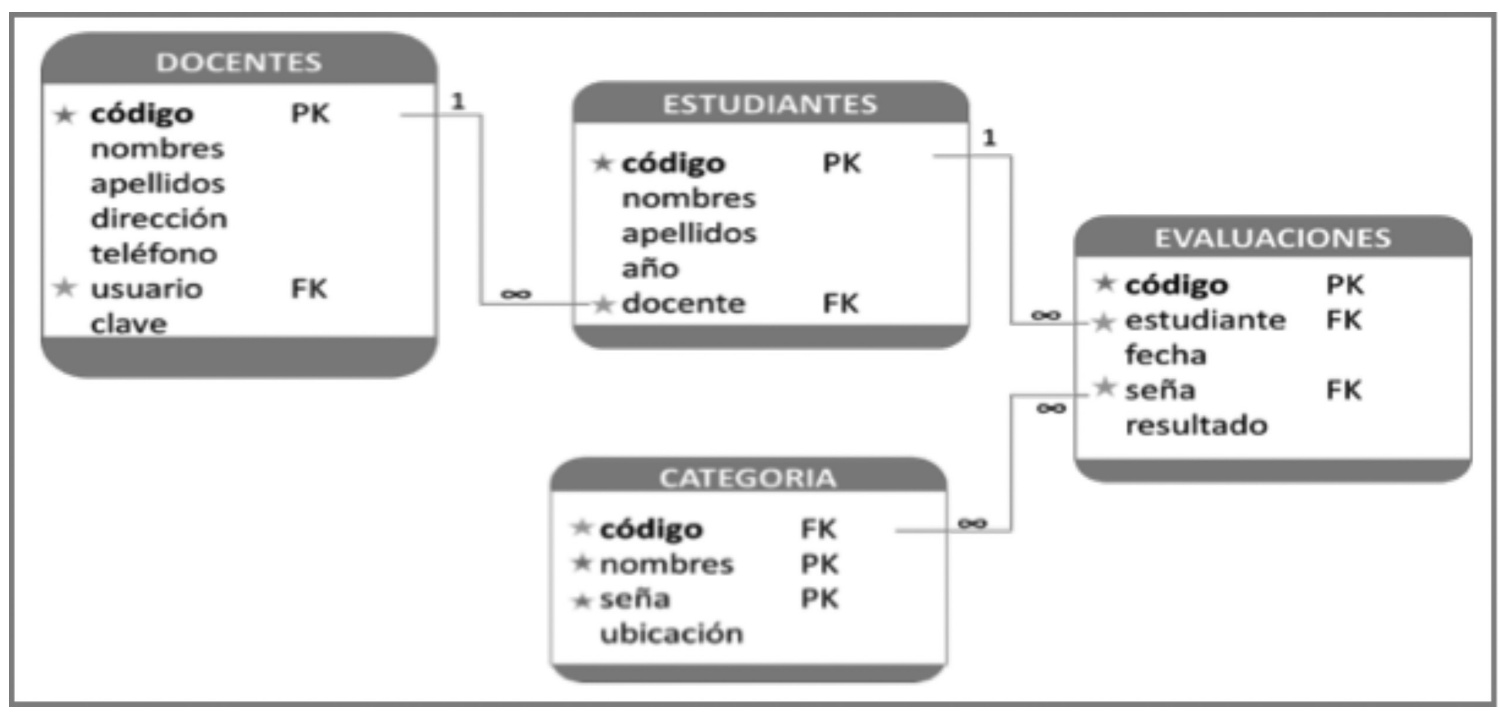

Fuente: Autores

Figura 4. Diagrama de secuencia

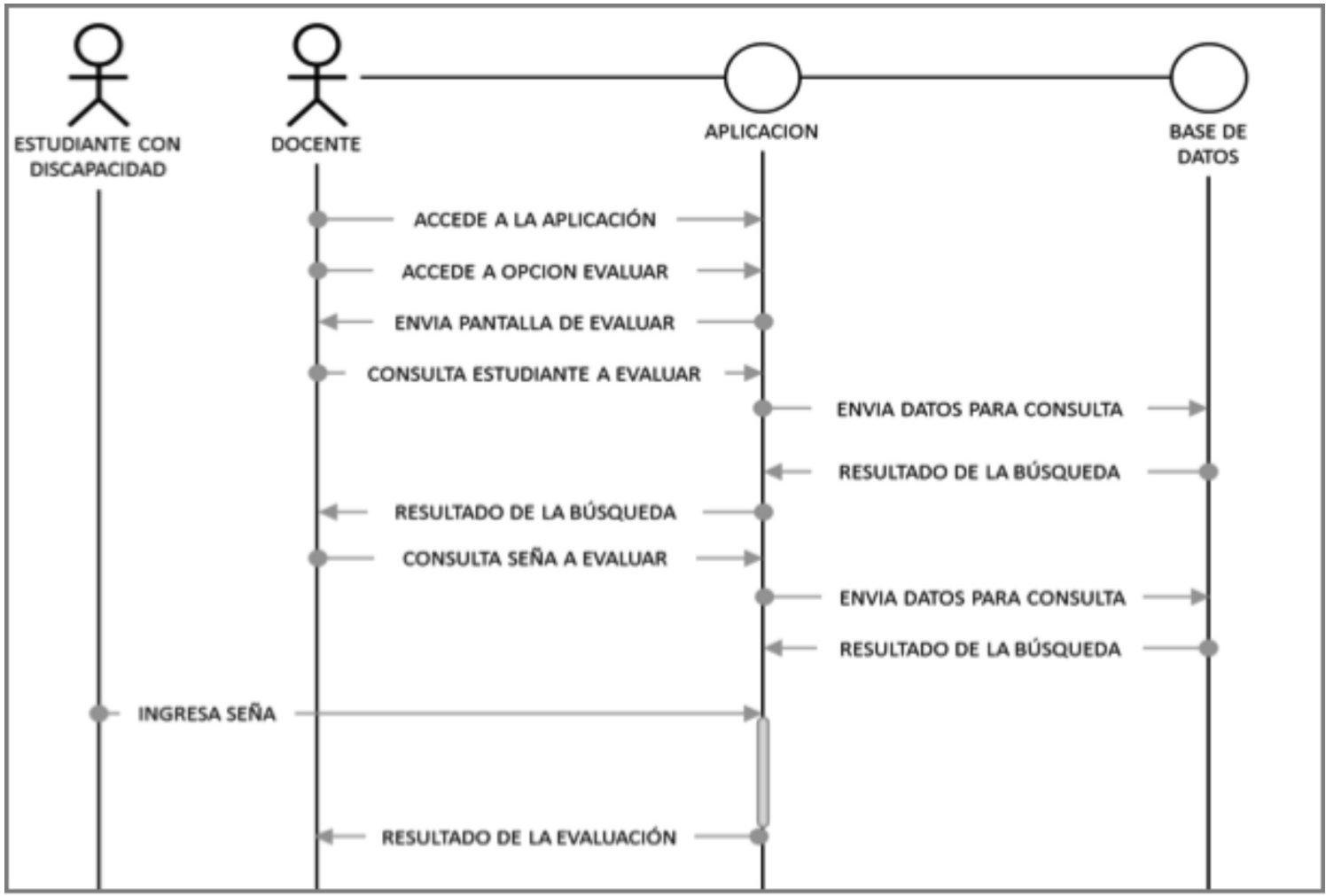

\section{DESARROLLO DEL SISTEMA}

Para el desarrollo del producto, se hizo uso de la tecnología que ofrece el Sensor Kinect para Xbox (ver Figura 5), el cual, permite a los usuarios controlar e interactuar con la consola sin ne- cesidad de tener contacto físico con un controlador de videojuegos tradicional, mediante una interfaz natural de usuario que reconoce gestos, comandos de voz, y objetos e imágenes. 
Tabla 2. Niveles de prueba

\begin{tabular}{|c|c|c|}
\hline \multicolumn{3}{|c|}{ Niveles de Pruebas } \\
\hline Test & Objetivo & Método \\
\hline Unitario & $\begin{array}{c}\text { Detectar errores en los datos, lógica, } \\
\text { algoritmos }\end{array}$ & Caja blanca \\
\hline Integración & $\begin{array}{l}\text { Detectar errores de interfaces y } \\
\text { relaciones entre componentes }\end{array}$ & Caja blanca \\
\hline Funcional & $\begin{array}{c}\text { Detectar errores en la implementación } \\
\text { de requerimientos }\end{array}$ & Caja negra \\
\hline Sistema & $\begin{array}{c}\text { Detectar fallas en el cubrimiento de los } \\
\text { requisitos }\end{array}$ & Caja negra \\
\hline Aceptación & $\begin{array}{l}\text { Detectar fallas en la implementación del } \\
\text { sistema }\end{array}$ & Caja negra \\
\hline
\end{tabular}

Fuente: Autores

Figura 5. Sensor Kinect para Xbox 360

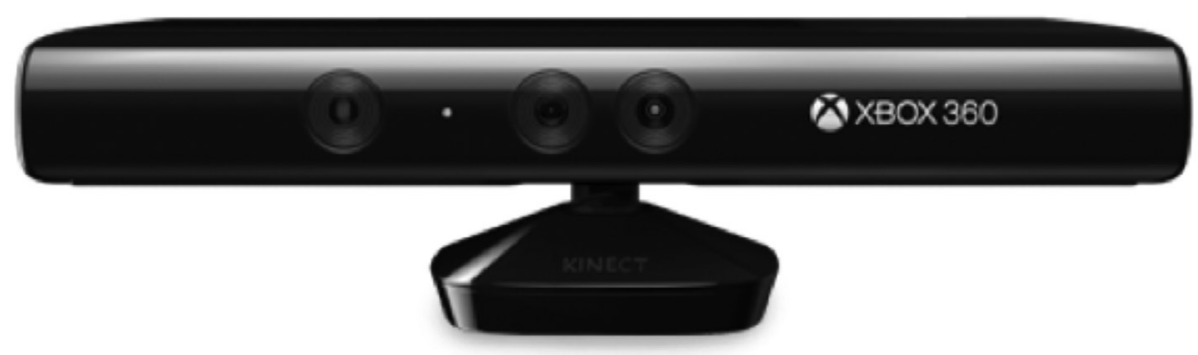

Fuente: (Khoshelham, 2012)

Haciendo uso de las ventajas que ofrece el Kinect se logró el objetivo principal de este proyecto en cuanto a reconocimiento e interpretación de algunos gestos de la lengua de señas colombiana, lo cual es un gran aporte a los niños que presentan discapacidad auditiva y que no cuentan con suficientes herramientas didácticas para aprender las señas en las instituciones educativas.

El Kinect cuenta con componentes que fueron vitales en el desarrollo del proyecto, como lo son la cámara de profundidad y el sensor infrarrojo; gracias a los cuales se logró el resultado esperado, que fue capturar la seña realizada por el usuario para luego procesarla (comparación de la imagen capturada con la base de datos de plantillas de señas) obteniendo un porcentaje de similitud con el que se determina si el gesto fue realizado correctamente. Para llegar a este resultado, se dividió la solución en tres etapas: Cap- tura, Procesamiento e Interpretación, las cuales se explican a continuación.

\section{Captura}

Este dispositivo cuenta con una cámara RGB, un sensor de profundidad, un micrófono de múltiples matrices y un procesador personalizado que ejecuta el software patentado, que proporciona captura de movimiento de todo el cuerpo en 3D, reconocimiento facial y capacidades de reconocimiento de voz. El sensor de profundidad es un proyector de infrarrojos combinado con un sensor CMOS monocromo que permite al Kinect ver la habitación en 3D en cualquier condición de luz ambiental (ver Figura 6). El rango de detección de la profundidad del sensor es ajustable gracias al software de Kinect capaz de calibrar automáticamente el sensor. (Microsoft, 2010) 
Figura 6. Componentes del Sensor Kinect para Xbox 360

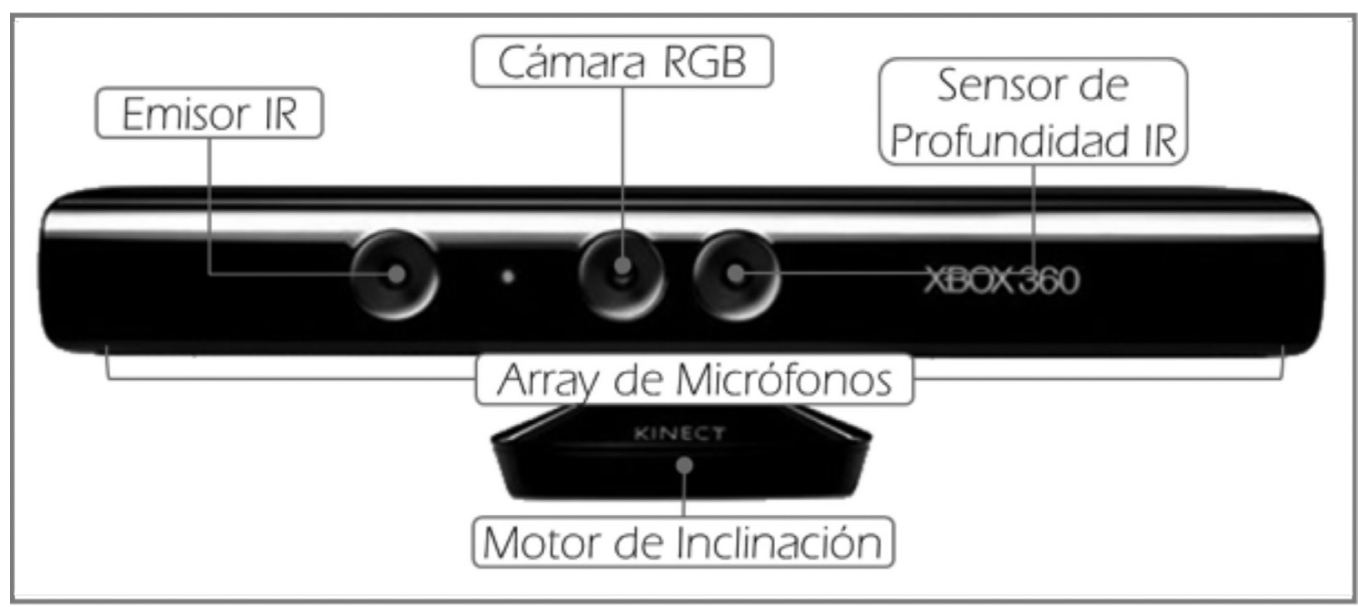

Fuente: (Khoshelham, 2012)

Gracias a estos componentes, se hace uso principalmente de la cámara RGB y el sensor de profundidad con dos resoluciones $(320 \times 240 \mathrm{y}$ $640 \times 480$ de alto color) y envían datos con una frecuencia de actualización de $30 \mathrm{fps}$, con los cuales se logró obtener una captura de la imagen de las manos a través de la interfaz diseñada mediante el lenguaje de programación c\# y la librería del SDK (Software development Kit) de Kinect (versión 1.8).

El proyector infrarrojo emite miles de puntos invisibles al ojo humano, pero gracias a la cámara infrarroja se pueden capturar dichos puntos que miden la distancia de los objetos ubicados frente al Kinect. A través de éstos, se obtiene un vector de bytes que contienen la información de la distancia de cada pixel de la imagen al sensor. Al tener 2 cámaras de infrarrojos cada píxel se corresponde con 2 bytes en el vector siendo éstos el valor de la distancia de ese píxel a cada cámara. La organización de los píxeles es la misma que con la cámara RGB, los 2 primeros bytes es la distancia del píxel de la posición de arriba a la izquierda al sensor y los 2 últimos son del píxel de abajo a la derecha (Perona, 2011) como se muestra en la Figura 7.

Figura 7. Organización de pixeles en el vector

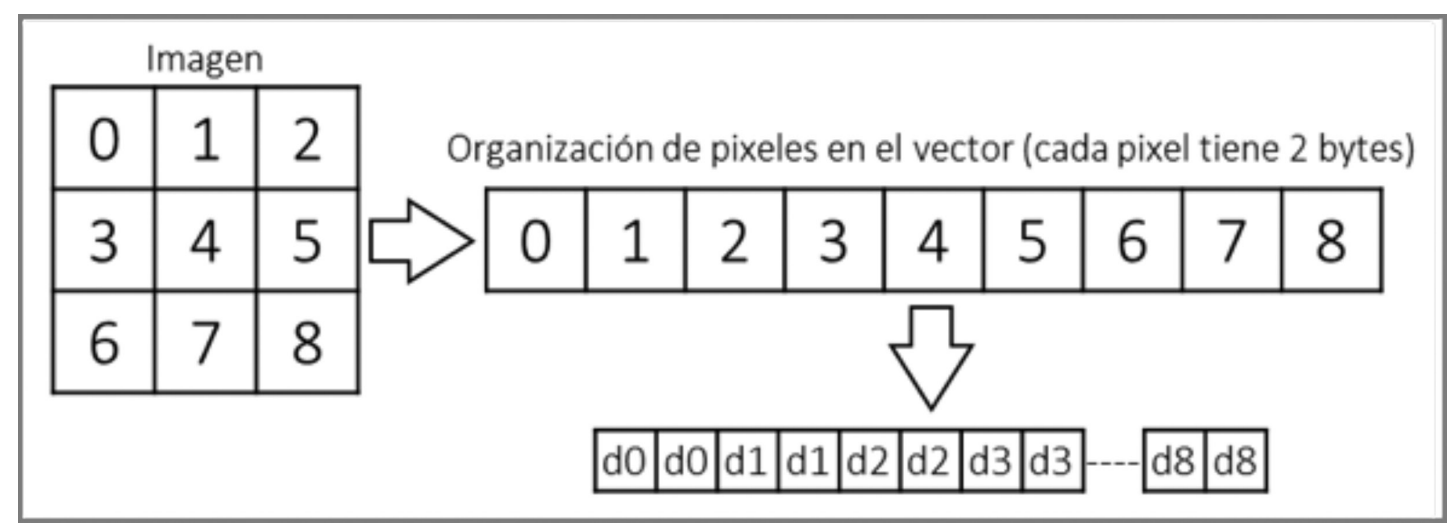


Con esta información, se identifican los pixeles más cercanos a la cámara, que de acuerdo a las diferentes pruebas realizadas, se determinó que para la óptima captura de los datos, la palma de la mano debe ubicarse frente al sensor a una distancia comprendida entre los $40 \mathrm{~cm}$ y máximo $1 \mathrm{~m}$ del campo de visión del dispositivo. Los puntos detectados son representados en pantalla en colores negro y blanco, siendo los negros los puntos más cercanos al sensor en la distancia establecida, es decir, la mano, dibujándose así la seña realizada como se muestra en la 13 , facilitándose el proceso de comparación con las plantillas, contrario a las imágenes RGB, las cuales presentan por cada pixel diferentes características de colores y variaciones de acuerdo el ambiente donde se realice la captura de la imagen.

\section{Procesamiento}

En esta fase se hace uso de la base de datos de plantillas, la cual se obtuvo de las capturas realizadas con el Kinect y está conformada por un conjunto de imágenes similares a la que se muestra en la Figura 8 con variaciones de tamaños de las manos y posiciones realizando la misma seña (ver Figura 9).

Figura 8. Captura de la mano realizada con Kinect

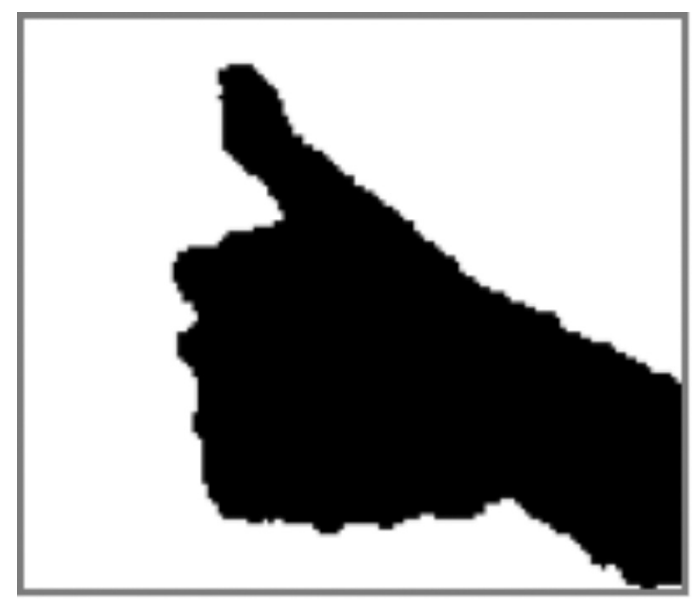

Fuente: (Khoshelham, 2012)

La imagen capturada se calibró de tal manera que se pudiera comparar con las plantillas de la seña seleccionada. Para esto se tuvo en cuenta el tamaño de la imagen el cual se configuró de 160x160 pixeles. Para realizar el procesamiento de la imagen, se implementó un método que recibe la captura realizada por medio de la aplicación y a partir de la seña que se desea evaluar, consulta en la base de datos las plantillas correspondientes.

Además, se diseñó un algoritmo de comparación el cual se ejecuta por cada plantilla existente en la base de datos, y recorre las imágenes pixel a pixel comparando los colores que las conforman a cada una (blanco y negro), por cada coincidencia encontrada se realiza un conteo a partir del cual se establece el valor promedio de pixeles iguales, y a partir de este valor se logra obtener el porcentaje de similitud entre la captura y el conjunto de plantillas.

\section{RESULTADOS}

A partir del porcentaje de similitud calculado en la fase de procesamiento, se determina finalmente si la seña fue realizada o no correctamente. El valor límite establecido para determinar si una seña fue realizada o no correctamente, se estipula luego de realizar varios experimentos donde se determina que si el porcentaje de similitud obtenido como resultado en la etapa de procesamiento es superior al 85\%, la aplicación indica que la seña fue realizada correctamente, de lo contrario, si se encuentra por debajo de este porcentaje, se indica que está incorrecta.

Gracias a las ventajas que ofrece el Kinect se logró el objetivo principal de este proyecto en cuanto a reconocimiento e interpretación de gestos de la lengua de señas colombiana, lo cual será un valioso aporte a la educación de los niños con discapacidad auditiva y que no cuentan con suficientes herramientas didácticas para aprender las señas.

Para el uso de la herramienta se debe programar el dispositivo, para que identifique la trayectoria de estos movimientos, y los asocie a su equivalente en el lenguaje escrito. Para esto, se limita el sistema al reconocimiento de una serie de signos. Luego de obtener estas dos bases, se procede a la lectura de movimientos, los cuales 
Figura 9. Base de Datos de Señas

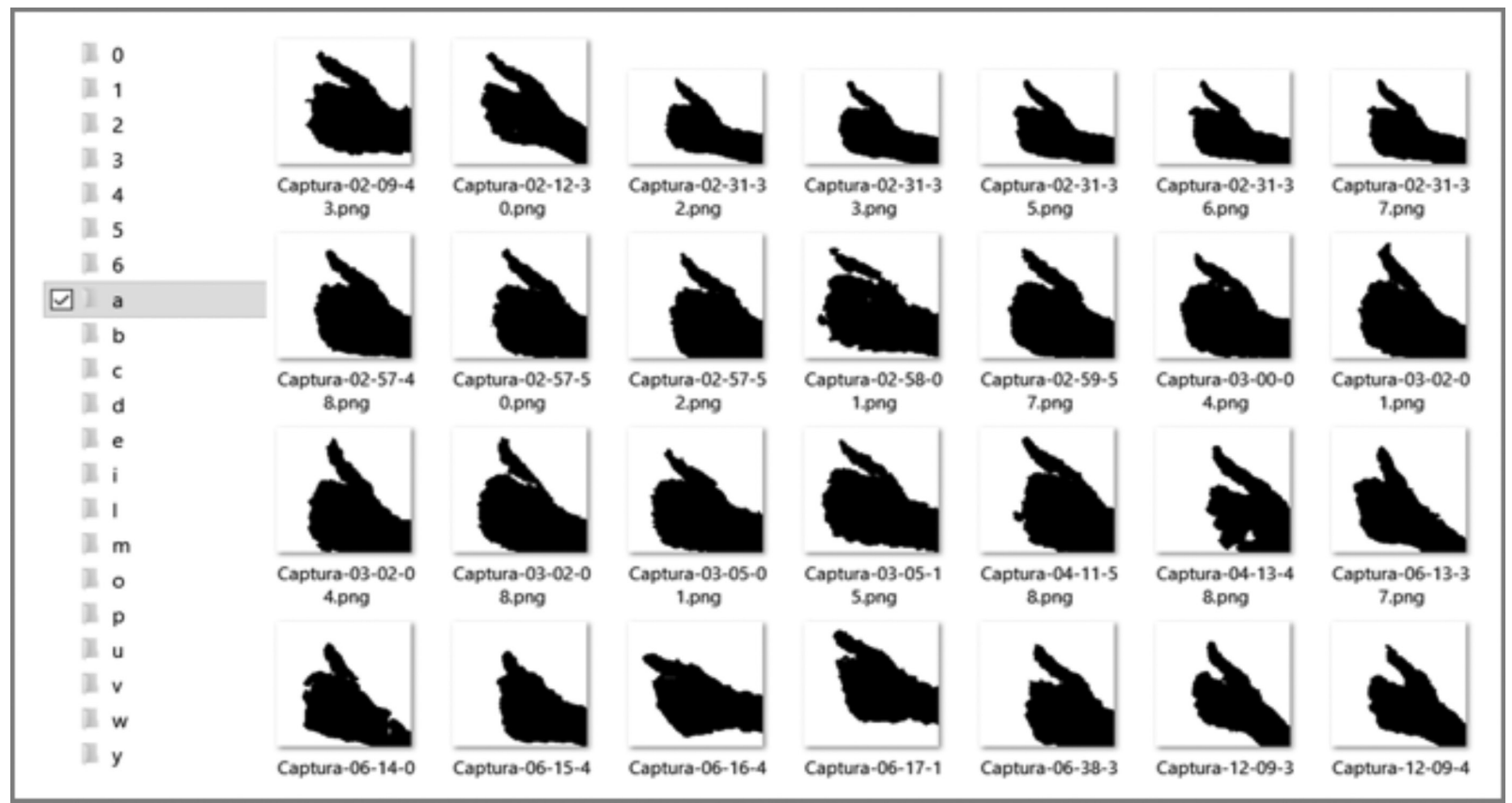

Fuente: Autores

deben ser interpretados, procesados, y mostrados en pantalla por medio de la interfaz de la aplicación, donde el usuario podrá visualizar la traducción de los gestos.

Por otra parte, para el enfoque académico que se ofrece, se mostrarán por medio de videos los gestos del lenguaje al aprendiz, quien será evaluado capturando de la misma forma por medio del Kinect.

Fragmentos de la herramienta informática se pueden observar en las figuras 10, 11,12 y 13.

Figura 10. Inicio sesión aplicación "Lengua de señas"

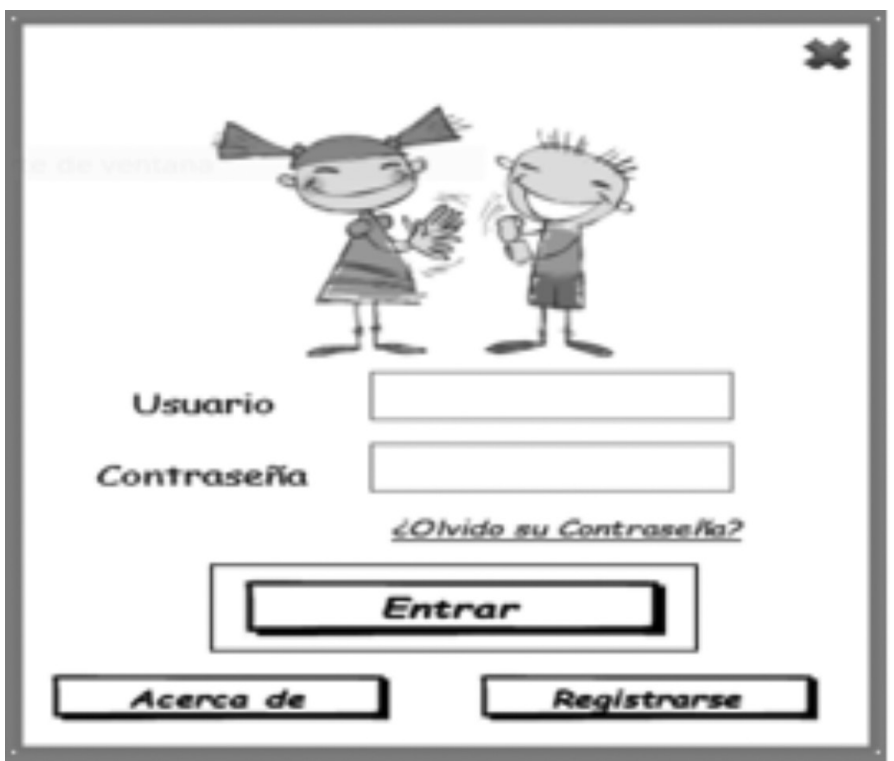

Fuente: Elaboración propia 
Figura 11. Menú principal aplicación “Lengua de señas”

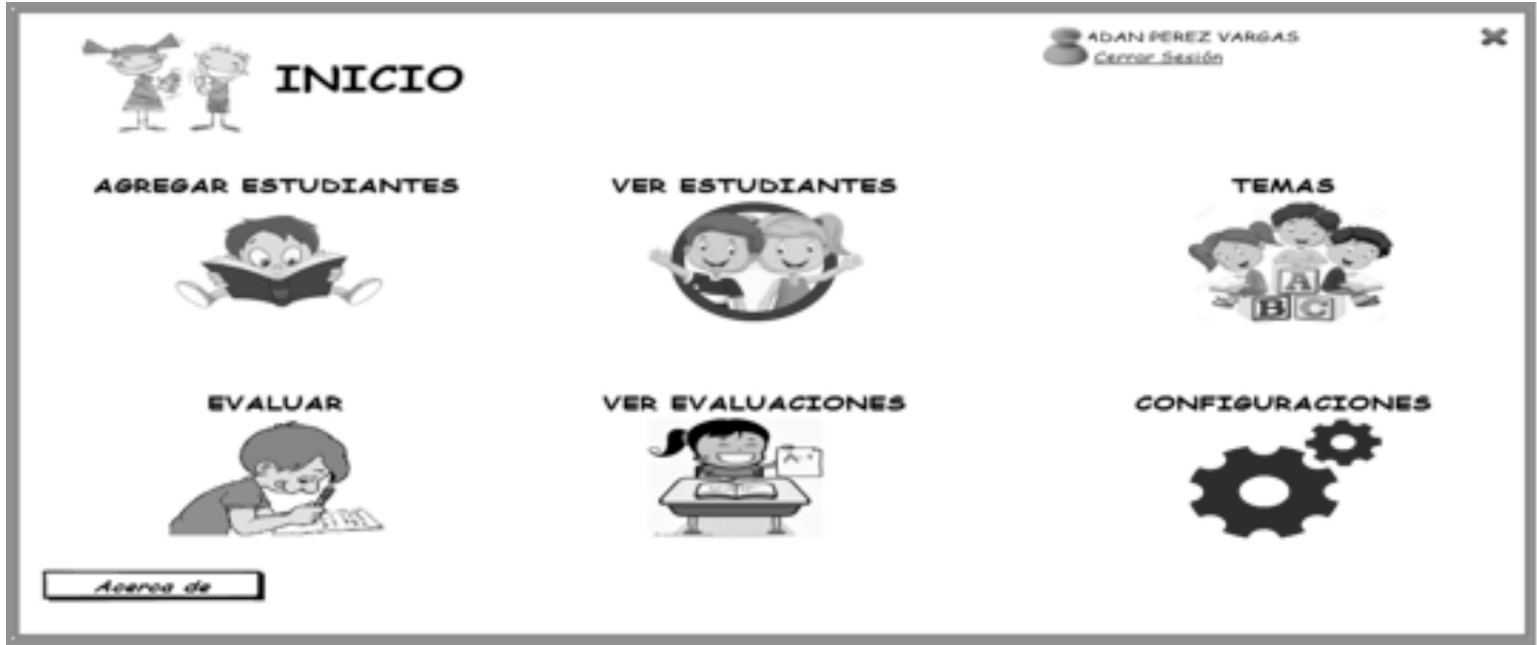

Figura 12. Evaluación aplicación "Lengua de Señas"

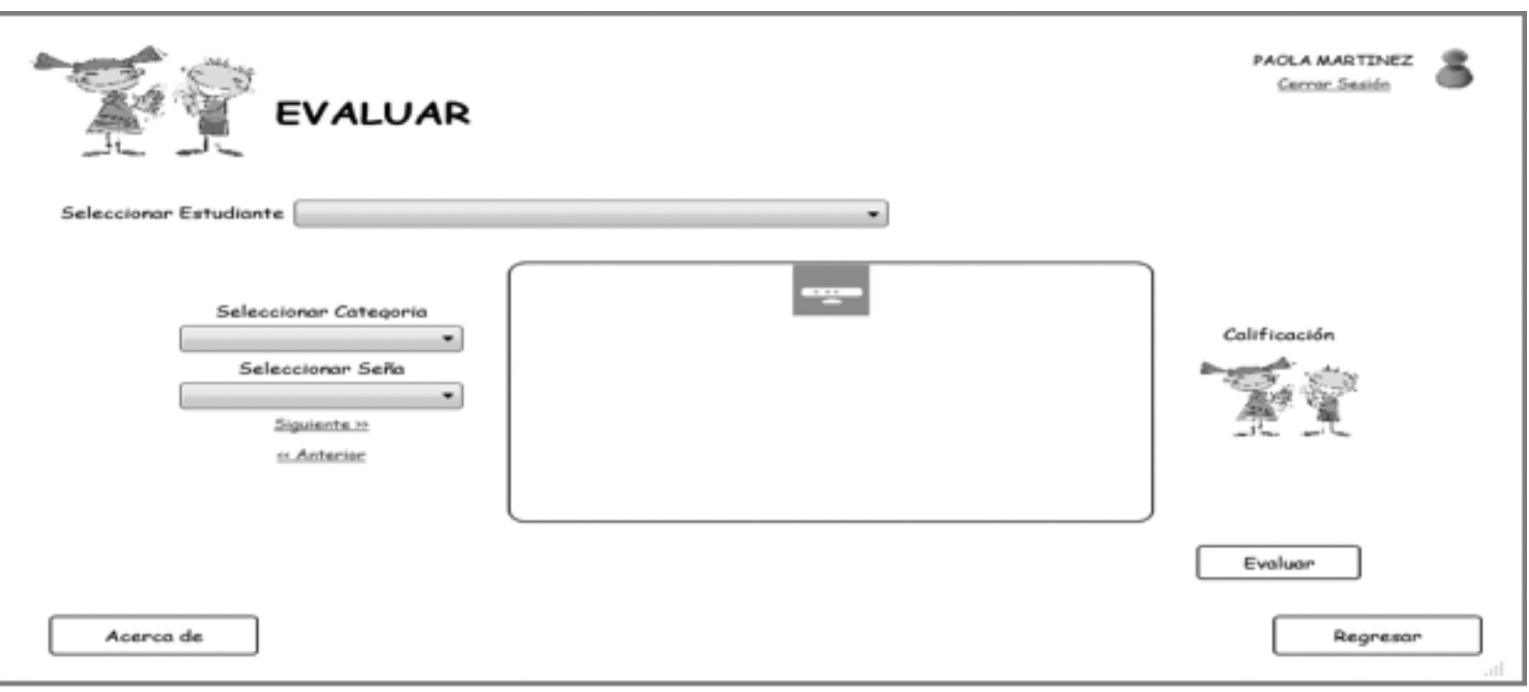

Figura 13. Selección de Temas.

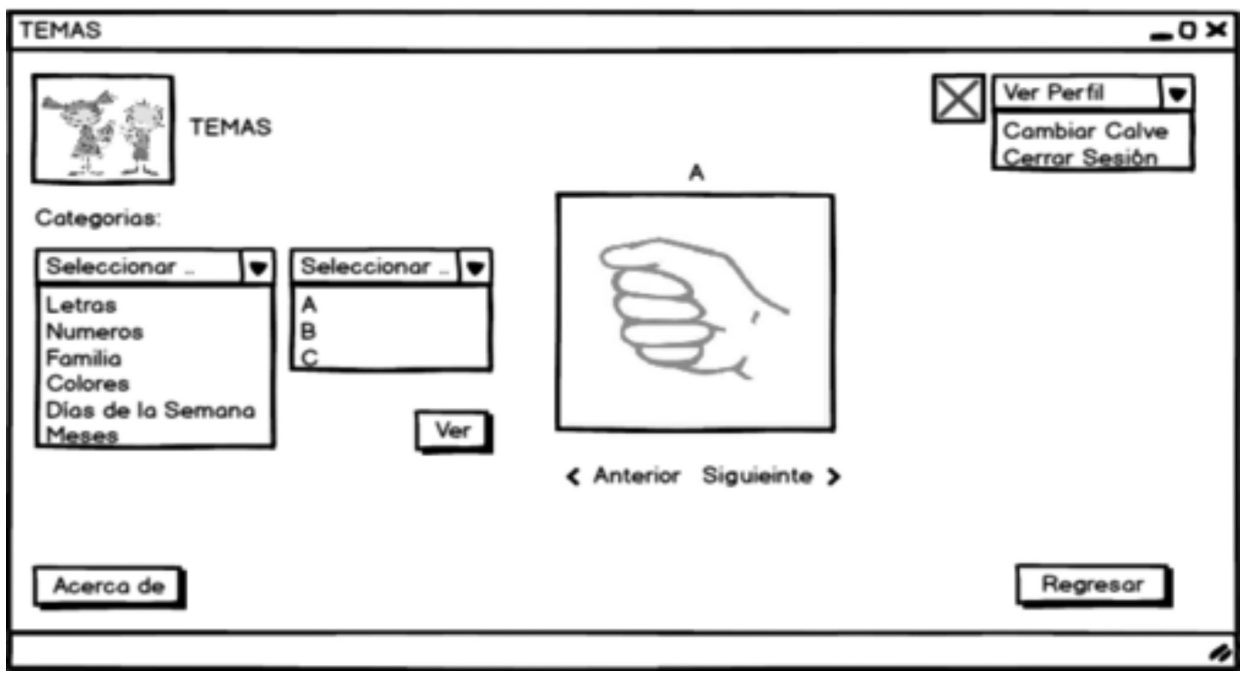




\section{Conclusiones}

- Haciendo uso de las ventajas que ofrece el Kinect se logró el objetivo principal de este proyecto en cuanto a reconocimiento e interpretación de algunos gestos de la lengua de señas colombiana, lo cual será un gran aporte a los niños que presentan discapacidad auditiva y que no cuentan con suficientes herramientas didácticas para aprender las señas.

- El Kinect cuenta con una cámara de profundidad y un sensor infrarrojo y gracias a esos componentes se logró el resultado esperado que fue capturar la seña realizada por el usuario para luego procesarla (comparación de la imagen capturada con la base de datos de imágenes) obteniendo un porcentaje de similitud con el que se determina si el gesto fue realizado correctamente.

- Una de las principales desventajas el Kinect es el manejo de la distancia del usuario que se encuentre ubicado dentro del campo de visión de este dispositivo, así como también las condiciones de iluminación y el ambiente. Por lo tanto se recomienda que para realizar la evaluación, la distancia apropiada de la mano este aproximadamente entre los $40 \mathrm{~cm}$ a 1 metro de distancia del Kinect y ubicarla de tal forma que en la cámara del software se logre captar solo la mano.

- Por otra parte, se recomienda usar la aplicación con el Kinect con óptimas condiciones de iluminación y sin objetos que puedan obstaculizar la cámara.

\section{Referencias}

Cujano, M. V., y Vera, C. I. (2016) Diseño e implementación de un sistema interactivo mediante tecnología Kinect v2.0 para desarrollar las habilidades psicomotrices en personas con discapacidad visual. Tesis de Ingeniería. Universidad Nacional de Chimborazo, 2016.

Castro, C. (2003) Aprendizaje del lenguaje en niños sordos: fundamentos para la adquisición temprana de lenguaje de señas [on line], 17 (1), p. 1-12. Disponible desde <http://www.psicologiacientifica.com/lenguaje-de-senas-aprendizaje / > [Acceso 27 de septiembre de 2016].
Constitucioncolombia. (1991). Constitución política de Colombia. Recuperado de < http:/ / www.constitucioncolombia.com/titulo-2/ capitulo-2/ articulo-67> [Acceso Noviembre 2016]

Khoshelham, K., \& Elberink, S. O. (2012). Accuracy and resolution of kinect depth data for indoor mapping applications. Sensors, 12(2), 1437-1454.

Ley324, (1996). Recuperado de http://www. alcaldiabogota.gov.co/sisjur/normas/Norma1. jsp?i=349 [Acceso Noviembre 2016]

López, E., Velásquez, J., Eleuterio, R., y Gil, L. (2015) Interfaz de reconocimiento de movimientos para el lenguaje de señas mexicano implementando el Kinect. Revista Aristas: Investigación Básica y Aplicada, 4(7), 130-133.

Ministerio de Educación Nacional. (2006). Educación Bilingüe para Sordos - Orientaciones Pedagógicas. Bogotá D.C, República de Colombia: Imprenta Nacional de Colombia. Obtenido de INSOR: Instituto Nacional Para Sordos: http:/ / www.insor.gov.co/historico/images / PUBLICACIONES/cartilla_etapa_escolar.pdf

Parra, J. A. (2015) Intérprete de lenguaje de signos para la comunicación de personas con discapacidad auditiva empleando procesamiento de imágenes. Rev. Invest. Univ. Quindío, 27 (1), 54-60.

Perona, J. (2011) Reto Kinect: Usar las cámaras del sensor [Internet], Disponible desde: <https://blogs.msdn.microsoft.com/ esmsdn / 2011 / 07 / 20 / reto-kinect-usar-las-cmaras-del-sensor/ > [Acceso 29 de agosto 2016]

Rodríguez, C., Pineda, J., y Sánchez, D. (2013) Prototipo traductor de señales manuales a texto legible, utilizando Kinect, AVANCES Investigación en Ingeniería, 10 (2), 64-72.

UN, (2006). Recuperado de <http://www. un.org/spanish / disabilities / convention/ convention.html $>$ [Acceso Noviembre 2016]

Villegas, O., y Barrientos, A. (2015) Propuestas de Soluciones TIC emergentes para Personas con Discapacidad. Sistemas, Cibernética e Informática., 12 (2), 1-6. 